Dieses Dokument ist eine Zweitveröffentlichung (Postprint) /

This is a self-archiving document (postprint):

Tobias Günther, Erich Querner, Rainer Groh

A head-in-hand metaphor for user-centric direct camera control in virtual reality

Erstveröffentlichung in / First published in:

MuC'20: Mensch und Computer, Magdeburg 6.-9.09.2020. ACM Digital Library, S. 129-132. ISBN 978-1-4503-7540-5

DOI: https://doi.org/10.1145/3404983.3410019

Diese Version ist verfügbar / This version is available on:

https://nbn-resolving.org/urn:nbn:de:bsz:14-qucosa2-748883 


\section{A head-in-hand metaphor for user-centric direct camera control in virtual reality}

\author{
Tobias Günther \\ tobias.guenther2@tu-dresden.de \\ Technische Universität Dresden \\ Dresden
}

\author{
Erich Querner \\ erichquerner@posteo.org \\ Technische Universität Dresden \\ Dresden
}

\author{
Rainer Groh \\ rainer.groh@tu-dresden.de \\ Technische Universität Dresden \\ Dresden
}

\begin{abstract}
The explorative examination of constructed 3D models in immersive environments requires suitable user-centric interaction methods. Especially novel concepts for virtual camera control can offer advantages, e.g. for the analysis of model details. We extend the known concept of the camera-in-hand metaphor and implement a multidimensional viewport control technique that can be used with common head-mounted displays and VR-controllers. With our head-in-hand view the user is able to control the virtual camera directly by hand without losing the flexibility of head movements. To ensure convenient operation, the method restricts special rotation parameters and smoothes jerky gestures of the user hand. In addition, we discuss implications and improvement potential of the proposed concept as well as adverse effects on the user, such as motion sickness.
\end{abstract}

\section{CCS CONCEPTS}

- Human-centered computing $\rightarrow$ Virtual reality; Gestural input.

\section{KEYWORDS}

Virtual Reality, 3D-interaction, camera control, user-centric, headin-hand metaphor

\section{ACM Reference Format:}

Tobias Günther, Erich Querner, and Rainer Groh. 2020. A head-in-hand metaphor for user-centric direct camera control in virtual reality. In Mensch und Computer 2020 (MuC'20), September 6-9, 2020, Magdeburg, Germany. ACM, New York, NY, USA, 4 pages. https://doi.org/10.1145/3404983.3410019

\section{INTRODUCTION}

The viewing and evaluation of 3D models is an important aspect of the working world. In automotive industry, Virtual Reality (VR) technologies enable the immersive analysis of structural elements, components and models. A key advantage is the realistic, true-toscale representation, which promotes the imagination of developers. Thus, engineers can compare and evaluate variants and concepts easily [19].

However, many VR applications do not fully exploit the potential of virtual phenomena. The limitations result from the desire to mirror reality and adopt various aspects of human perception. Nevertheless, virtual worlds offer a large number of visualization

MuC'20, September 6-9, 2020, Magdeburg, Germany

(C) 2020 Copyright held by the owner/author(s). Publication rights licensed to ACM

This is the author's version of the work. It is posted here for your personal use. Not for redistribution. The definitive Version of Record was published in Mensch und Computer 2020 (MuC'20), September 6-9, 2020, Magdeburg, Germany, https://doi.org/10.1145/ 3404983.3410019 . and interaction possibilities beyond the laws of nature. Examples are superpowers like teleporting, X-ray vision or shrinking the user. We think those ideas can be beneficial for VR applications.

When the user evaluates 3D models on a normal desktop system, the viewing position is static most of the time. Instead of changing the own body position to obtain different perspectives on the object, the user probably rotates, scales and moves the model itself. The view is object-centric [14]. In immersive environments it mostly depends on the size of the object. The user can grab small objects with the hands or the controllers to observe them. But the handling of larger models is clunky and not convenient. In most cases an appropriate way for the user is to walk around the 3D model and change his or her own position to get the desired view. The view is user-centric [14], the object is static and maintains its natural size and orientation.
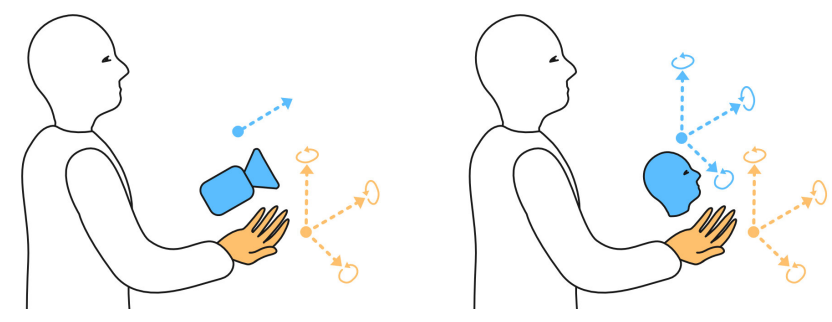

Figure 1: Camera-in-hand metaphor (left): the viewport is completely linked to position and orientation of the hand. Head-in-hand metaphor (right): the user is also able to influence the viewport orientation with his head movements.

Problems arise from the following scenario: the user wants to analyse even tiny details of a complex 3D model. With normal view in VR this can be difficult and physically demanding. Furthermore, there may be contorted rooms or niches that are not accessible for the view. We think there are many uses for a kind of zooming camera that can be held in the hand to adequately view small details and reach otherwise inaccessible places. To ensure broad applicability and to make the method available to small and mediumsized companies as well as private users, we only use common and accessible VR hardware: a standard head-mounted display and associated controllers. Our goal is to transfer the well known camera-in-hand metaphor to a modern VR environment and extend it to a head-in-hand metaphor (see Fig. 1).

\section{RELATED WORK}

A mental precursor of the camera-in-hand metaphor is the endoscopy. The idea goes back to ancient times, when doctors used 
mirror constructions to look into easily accessible body orifices. The first optical endoscopes with light guides for illumination and reflection guidance to the observing eye were already developed in the 19th century [6]. Brubaker and Holinger first combined an endoscope and a video camera in 1941 [10]. Live feedback via television emerged in the 1950s, as well as flexible endoscopes [6]. The technical possibility to control your own view with the movements of your hand in real time arose during this time. Other previous technical devices include hand-held video cameras, which appeared as early as the end of the 19th century [1]. The first hand-held cameras that offered live broadcasting did not enter the market until the 1960s [2].

The concept of gaze control by hand for viewing virtual environments could only emerge with the rise of computer graphics and 3D tracking devices. The first 3D input device was created in 1963 [21]. After further technical improvements in the 1970s [11], first considerations were made in the 1980 s to link the spatial position and orientation of the hand with the virtual camera image $[4,8,9]$.

\subsection{Camera-in-hand metaphor (non-immersive)}

Badler et al. use a six degree-of-freedom sensor to change the position and orientation of the viewport [4]. The virtual 3D scene contains only few rectangular blocks and is displayed in real time on a CRT display. A problem is the missing of spatial feedback, because the used display is neither stereoscopic nor immersive. Furthermore, the simple scene offers hardly any depth cues for orientation. The authors note that absolute positioning is easier to control than relative positioning. Absolute means the direct transmission of the hand movement to the camera movement. For relative movement, users press a button when they wish to transmit the hand movement. Whenever the button is released, the position and orientation of the camera remains static. The advantage is that the user does not have to stay in uncomfortable positions to point the virtual camera at the scene. Otherwise, the complicated orientation has an unfavourable effect.

In literature the "camera-in-hand" metaphor is synonym to "eyeballin-hand". This term is first introduced by Brooks. In his work, the eyeball is controlled by a six degree-of-freedom sensor to perform architectural walkthroughs $[8,9]$. Brooks describes this method as "ideal for map navigation but quite confusing for scene navigation" [9]. A disorientation factor can be suspected in the hardware used at the time. The scene was not presented in real time.

A fundamental publication appeared in 1990: Ware and Osborne compare three different metaphors for exploring the virtual environment and controlling the virtual viewport [23]. A six degreeof-freedom input device is used. They introduce the following metaphors: "eyeball-in-hand", "scene-in-hand" and "flying vehicle control". The first one is explained as a possibility to steer a virtual camera by hand to view the virtual scene. In reality the user holds the input device in his hand and orbits an invisible model which he or she can only see on the monitor. The authors discover that this method is easy to learn and understand.

Analogous to Balder et al., the authors test absolute and relative transmission of movements. They decide on the absolute variant because otherwise the mental model of the users is damaged and users can no longer imagine the fixed invisible scene. The authors mention in particular physical limitations of the setup with regard to the tasks to be solved by test persons. The tracked space of the input device is too small to view larger scenes. In addition, the low resolution of the device makes it difficult to view an object without camera shake. Subjects dislike physical contortions and the effort to view a whole scene, since they must walk a lot to view all objects.

To overcome the weaknesses of the isolated use of the camerain-hand metaphor, further concepts were proposed, combining for example different metaphors, motion mappings or bimanual camera control $[5,20]$. De Boeck and Coninx complement the camera-inhand metaphor with a flying-vehicle metaphor [13]. The virtual camera is placed onto the vehicle. This vehicle is controlled by moving the input sensor in a borderline area. The PHANToM system is used as sensor, which can offer the user haptic feedback. Depending on the direction and pressure with which the sensor is moved, the vehicle can be controlled. As long as the user moves within the limits of the borderline area, only the position and rotation of the virtual camera is changed. As soon as the user pushes against the limits, the vehicle on which the camera is standing also moves.

\subsection{Camera-in-hand metaphor (immersive)}

So far, the approaches use non-immersive displays for showing the virtual scene to the users. In the following we summarize papers which utilize the camera-in-hand metaphor in conjunction with head-mounted displays.

Stoev et al. present various techniques for navigation in virtual environments, all of which are displayed in an additional virtual window [22]. The method is called "through-the-lens" concept. The actual viewing of the scene takes place, as usual in virtual reality, through the natural movement and head tracking of the user. The viewport is thus controlled by the user's position and head movements. The through-the-lens window is displayed in the user's field of view and overlays a part of the actual scene. One mode features a camera-in-hand view. The window is moved with one hand and the additional virtual camera with a pen in the other hand. According to the authors, deficits of disorientation can be overcome in this way. The feedback of the camera change takes place without delay, and the user keeps the overview through the external view of the virtual scene.

Another work of Fukatsu et al. proposes a manipulation method to control multiple viewports in immersive environments [15]. The authors couple the movement of an additional viewport with the relative movement of the primary viewpoint. The user can control both viewports with his head and hand movements. The concept is similar to that of Stoev et al. Again, the additional viewport is displayed in an extra window within the user's immersive view. The idea is compared to classic eyeball-in-hand and scene-in-hand concepts. The implementation of the eyeball-in-hand variant does not use the advantages of the tracked user head. The viewport is static in the experiments. Therefore it is not surprising that the approach has the same deficits as previous work with nonimmersive displays. 


\subsection{Predecessors of the head-in-hand metaphor}

As explained, Badler et al. use a six degree-of-freedom sensor to control the virtual viewport. Because this device has the form of a stylus, they call it "wand". They describe an interesting metaphor: The displayed perspective is what "an observer sitting in the wand and looking down its long axis would see" [4]. This idea of a shrunken user inside of the tracked device is already near the concept of holding yourself in your own hand to reach small contorted spaces. The mental step to the head-in-hand metaphor is not far away. Surprisingly, we could not find any works that implement the concept. Although there are descriptions of hardware setups in the literature that would have easily allowed this method, the authors did not decide to implement it $[15,22]$.

We want to note, that there is an enormous amount of other methods to control the virtual viewport by means of the hands, like hand-directed steering techniques where the key element is the "continuous control of direction" [17] or gesture controlled locomotion techniques [7, 12]. An overview of the methods was compiled by $\mathrm{Al}$ Zayer et al. [3].

\section{METHOD}

In the following sections we outline the concept and implementation of the head-in-hand metaphor. As mentioned in related works section, there are already approaches of immersive camera-in-hand metaphors. The problem of the method of Fukatsu et al. is the fixed setup. In their experiments, the user is forced to be in camera-inhand mode the whole time. He or she is not able to switch to walking mode. On top of that, the head tracking of the head-mounted display is not applied. The only way to move the viewport is to change the position and rotation of the hand.

The work of Stoev et al. is more interesting for our use case. The user keeps the orientation over the scene. In addition, head tracking is used to some extend. At least the user is able to walk and navigate the virtual environment in standard way with firstperson view. Head tracking is not linked with the camera-in-hand view. Furthermore, the additional window is quite small. Thus, it is hard to see details of models. This is relevant with regard to the resolution of current head-mounted displays. In addition, the two-handed handling with window and pointer appears cumbersome and error-prone. Stoev et al. do not describe any method of smoothing out inaccuracies of hand movement such as trembling. It can be assumed that the output of the virtual handheld camera is shaky when the viewport is moved.

The transfer of the camera-in-hand metaphor to an immersive scenario with room-scale VR allows to eliminate many deficits of previous non-immersive approaches. We intend the head-inhand mode as an additional viewing variant that users can activate if required. This reduces problems with disorientation. The user can explore the scene in the VR application as usual by walking through it. The viewport remains linked to his position and head movements. Only if necessary, the user should switch to head-inhand mode. Even then, head movements are available like before, only the position is changed to the hand of the user and the viewport appears reduced in size. The virtual scene of the prototype contains a skybox with horizon, a textured floor and some 3D-models.

\subsection{Head-in-hand metaphor}

In our first implementation we realized the head-in-hand mode as a pure viewport without additional visual cues. However, the missing visual reference caused a loss of orientation. The users want to see their own hands, a pointer or at least a clearly perceptible change between states. We added a kind of glass shell with struts as pointer (see Fig. 2). The idea is to use the concept of rest frames for the pointing tool. Rest frames can reduce motion sickness in VR applications [16]. This is based on the observation that people have a better perception of resting objects. Rest frames represent an artificial fixed point to which the user can orientate himself. When in head-in-hand mode, our pointing glass shell tool is displayed around the user and is fixed to the viewport. Some users describe the impression as similar to sitting in a small spaceship. We introduced another change to promote the mental model: The user must first grab the pointer from the workbench using the VR controller and can then switch to the interior view at the touch of a button.
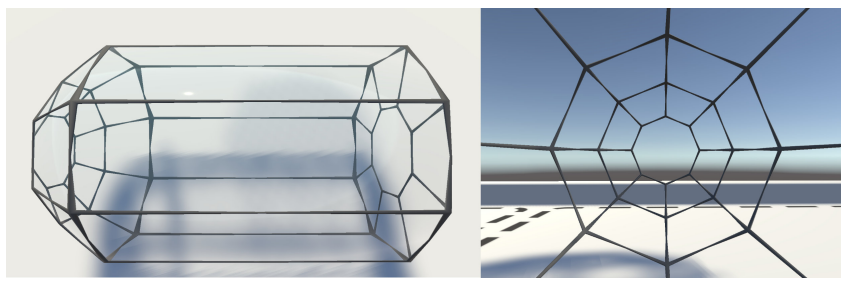

Figure 2: Pointing tool for the head-in-hand mode. Outside appearance (left) and look from the inside (right).

Keeping the hand still in one place for a long period of time is particularly strenuous. As already described in other works, the head-in-hand view is not suitable for slow and longer explorations. To counteract the disadvantages of the camera-in-hand metaphor described in related work, we implement two improvements: The restriction of the rotation axes and the use of a smoothing algorithm to compensate for camera shake and restless hand movements.

The restriction of rotation axes refers to the transfer of hand movements to the orientation of the viewport. Especially rolling movements pose a problem (i.e. rotations along the longitudinal axis of the view). These rotations make the horizon appear crooked, which can be uncomfortable for the user and make orientation difficult. In our tests it was in almost all cases the best solution to completely prevent such rotations. In contrast, yaw rotations must remain allowed. These are most familiar to the user, and correspond to looking around along the horizon line. Regarding pitch rotations (up and down rotations), the opinions of the users were not clear. Some preferred to turn off or at least reduce the intensity of pitch rotations. Others found the flexibility advantageous. We want to mention that the user still has the possibility to perform all rotations by natural head movement, only the transmission of hand orientation is limited. The user can press the touchpad of the VR controller to set different intensities of smoothing for hand movements. Smoothing works by averaging over tracking points. In our case, the last two determined hand positions and orientations are used for the calculation. 


\section{DISCUSSION AND FUTURE DIRECTIONS}

The visual appearance of the pointing device was criticised by users. The cobweb-like structure blocks the field of view too much. We still think that the use of rest frames is useful to give users a visual anchor in the head-in-hand view. However, the rest frame should be more discreet and possibly provide a floor and some reference point for comparison with the current user size. Users should notice their smaller size and thus have the possibility to get closer to details.

Despite efforts to reduce discomfort and disorientation, users may experience motion sickness. Smoothing the hand movements could counteract this to a certain degree. However, high smoothing factors gave the impression of latency or "trailing" movements. The restriction of movement axes or hand rotations is also a suitable feature to counteract motion sickness. On the one hand, this procedure is less demanding on the user. On the other hand, it is an abstraction of the movements. A compromise solution seems appropriate.

The way of smoothing viewport movements has a crucial influence on the user experience. One direction for the future is to evaluate different smoothing algorithms and intensities. How do the parameters need to be adjusted to provide the optimal experience? Other approaches for user studies deal with the metaphor itself. We aim to compare the method with similar techniques: traditional camera-in-hand, through the lens, magnifying glasses. Another promising approach is the use of transitions between viewport changes [18]. We think, transitions are able to facilitate the mental link between the different view modes.

\section{CONCLUSION}

We extended the camera-in-hand methaphor to a head-in-hand metaphor and implemented a prototype in an immersive environment to show the viability of our concept. With this approach the user is able to control the virtual camera directly by hand without losing the flexibility of head movements. The resulting tool allows the explorative examination of 3D models and especially small details and otherwise inaccessible corners. The technique can be used with standard head-mounted displays and VR-controllers. In contrast to the head movements of the user, rotation axis restrictions of the hand movements are activatable to prevent disorientation and a inconvenient operation. Additionally, a smoothing algorithm was implemented, which smoothes jerky gestures of the user hand.

\section{ACKNOWLEDGMENTS}

The authors gratefully acknowledge the Sächsische Aufbaubank (SAB), Germany and the European Social Fund (ESF) for their sponsorship of this research.

\section{REFERENCES}

[1] Richard Abel. 2005. Encyclopedia of early cinema. Routledge, Abingdon, Oxon, UK \& New York, USA

[2] Albert Abramson. 2003. The history of television, 1942 to 2000. McFarland \& Company, Inc., Jefferson, North Carolina, USA.

[3] Majed Al Zayer, Paul MacNeilage, and Eelke Folmer. 2020. Virtual Locomotion A Survey. IEEE Transactions on Visualization and Computer Graphics 26, 6 (2020), 2315-2334. https://doi.org/10.1109/TVCG.2018.2887379

[4] Norman I. Badler, Kamran H. Manoochehri, and David Baraff. 1987. MultiDimensional Input Techniques and Articulated Figure Positioning by Multiple Constraints. In Proceedings of the 1986 Workshop on Interactive 3D Graphics
(Chapel Hill, North Carolina, USA) (I3D '86). Association for Computing Machinery, New York, NY, USA, 151-169. https://doi.org/10.1145/319120.319132

[5] Ravin Balakrishnan and Gordon Kurtenbach. 1999. Exploring Bimanual Camera Control and Object Manipulation in 3D Graphics Interfaces. In Proceedings of the SIGCHI Conference on Human Factors in Computing Systems (Pittsburgh, Pennsylvania, USA) (CHI '99). Association for Computing Machinery, New York, NY, USA, 56-62. https://doi.org/10.1145/302979.302991

[6] George Berci and Kenneth A. Forde. 2000. History of endoscopy. Surgical endoscopy 14, 1 (2000), 5. https://doi.org/10.1007/s004649900002

[7] Doug Bowman, Chadwick Wingrave, Joshua Campbell, and Vinh Ly. 2001. Using Pinch Gloves(TM) for both Natural and Abstract Interaction Techniques in Virtual Environments. Technical Report TR-01-23. Computer Science, Virginia Tech.

[8] Frederick P. Brooks. 1987. Walkthrough-a Dynamic Graphics System for Simulating Virtual Buildings. In Proceedings of the 1986 Workshop on Interactive 3D Graphics (Chapel Hill, North Carolina, USA) (I3D '86). Association for Computing Machinery, New York, NY, USA, 9-21. https://doi.org/10.1145/319120.319122

[9] F. P. Brooks. 1988. Grasping Reality through Illusion-Interactive Graphics Serving Science. In Proceedings of the SIGCHI Conference on Human Factors in Computing Systems (Washington, D.C., USA) (CHI '88). Association for Computing Machinery, New York, NY, USA, 1-11. https://doi.org/10.1145/57167.57168

[10] J. D. Brubaker and Paul H. Holinger. 1941. The larynx, bronchi and esophagus in Kodachrome. The fournal of the Biological Photographic Association 2, 10 (1941), 83-91.

[11] Robert P. Burton and Ivan E. Sutherland. 1974. Twinkle Box: A Three-Dimensional Computer Input Device. In Proceedings of the May 6-10, 1974, National Computer Conference and Exposition (Chicago, Illinois) (AFIPS '74). Association for Computing Machinery, New York, NY, USA, 513-520. https://doi.org/10.1145/1500175. 1500278

[12] Marcio C. Cabral, Carlos H. Morimoto, and Marcelo K. Zuffo. 2005. On the Usability of Gesture Interfaces in Virtual Reality Environments. In Proceedings of the 2005 Latin American Conference on Human-Computer Interaction (Cuernavaca, Mexico) (CLIHC '05). Association for Computing Machinery, New York, NY, USA, 100-108. https://doi.org/10.1145/1111360.1111370

[13] Joan De Boeck and Karin Coninx. 2002. Haptic Camera Manipulation: Extending the Camera in hand Metaphor. In Proceedings of Eurohaptics 2002. Edinburgh, UK, $36-40$.

[14] Joan De Boeck, Chris Raymaekers, and Karin Coninx. 2005. Are existing metaphors in virtual environments suitable for haptic interaction. In Proceedings of 7th International Conference on Virtual Reality (VRIC 2005). 261-286.

[15] Shinji Fukatsu, Yoshifumi Kitamura, and Fumio Kishino. 2003. Manipulation of Viewpoints in 3D Environment using Interlocked Motion of Coordinate Pairs. In International Conf. on Human-Computer Interaction - INTERACT'03, Vol. 3. 327-334.

[16] Joseph J. LaViola. 2000. A Discussion of Cybersickness in Virtual Environments. SIGCHI Bull. 32, 1 (Jan. 2000), 47-56. https://doi.org/10.1145/333329.333344

[17] Joseph J. LaViola, Ernst Kruijff, Ryan P. McMahan, Doug Bowman, and Ivan P. Poupyrev. 2017. 3D User Interfaces: Theory and Practice (2nd ed.). Addison-Wesley, Boston. 624 pages.

[18] Kasra Rahimi Moghadam, Colin Banigan, and Eric D Ragan. 2020. Scene Transitions and Teleportation in Virtual Reality and the Implications for Spatial Awareness and Sickness. IEEE Transactions on Visualization and Computer Graphics 26, 6 (2020), 2273-2287. https://doi.org/10.1109/TVCG.2018.2884468

[19] Mathias Müller, Tobias Günther, Dietrich Kammer, Jan Wojdziak, Sebastian Lorenz, and Rainer Groh. 2016. Smart Prototyping-Improving the Evaluation of Design Concepts Using Virtual Reality. In International Conference on Virtual, Augmented and Mixed Reality, Stephanie Lackey and Randall Shumaker (Eds.). Springer International Publishing, Cham, 47-58. https://doi.org/10.1007/978-3319-39907-2_5

[20] Mahdi Nabiyouni, Bireswar Laha, and Doug A Bowman. 2014. Poster: Designing effective travel techniques with bare-hand interaction. In 2014 IEEE Symposium on 3D User Interfaces (3DUI). IEEE, 139-140. https://doi.org/10.1109/3DUI.2014. 6798859

[21] Lawrence G. Roberts. 1966. The Lincoln WAND. In Proceedings of the November 7-10, 1966, Fall foint Computer Conference (San Francisco, California) (AFIPS '66 (Fall)). Association for Computing Machinery, New York, NY, USA, 223-227. https://doi.org/10.1145/1464291.1464317

[22] Stanislav L. Stoev, Dieter Schmalstieg, and Wolfgang Straßer. 2001. Two-Handed Through-the-Lens-Techniques for Navigation in Virtual Environments. In Immersive Projection Technology and Virtual Environments 2001. Springer Vienna, 51-60. https://doi.org/10.1007/978-3-7091-6221-7 6

[23] Colin Ware and Steven Osborne. 1990. Exploration and Virtual Camera Control in Virtual Three Dimensional Environments. In Proceedings of the 1990 Symposium on Interactive 3D Graphics (Snowbird, Utah, USA) (I3D '90). Association for Computing Machinery, New York, NY, USA, 175-183. https: //doi.org/10.1145/91385.91442 